Abanico Veterinario. Enero-Diciembre 2021; 11:1-10. http://dx.doi.org/10.21929/abavet2021.2 Artículo Original. Recibido: 22/05/2020. Aceptado: 22/12/2020. Publicado: 04/01/2021. Clave: 2020-86.

\title{
Coccidiosis en pollos de engorda comerciales en Brazil entre 2012 y 2019: especies principales y grados de daño
}

\section{Coccidiosis in commercial broilers in Brazil between 2012 and 2019: main species and degrees of injury}

\section{Gazoni Fabio ${ }^{1 D^{\star}}$, Matte Fabrizio ${ }^{11 \mathrm{D}}$, Chiarelli-Adorno Felipe ${ }^{11 \mathrm{D}}$, Mariely- Jaguezeski Antonise 2ID, Tellez-Isaias Guillermo ${ }^{310}$, Schafer-da-Silva Aleksandro ${ }^{4 I D}$}

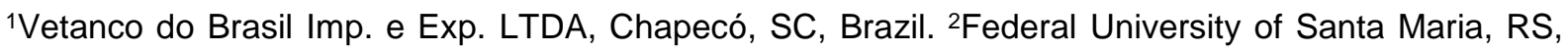
Brazil. ${ }^{3}$ Department of Poultry Science, University of Arkansas, Fayetteville, AR 72704, United States of America. ${ }^{4}$ Department of Animal Science, State University of Santa Catarina (UDESC), Chapecó, Brazil. *Autor para correspondencia: Rua Raimundo Zanella, 400D - Distrito Industrial Flávio Baldissera - CEP 89813-824. Chapecó, Brazil. gazoni@vetanco.com.br, fabrizio@vetanco.com.br, chiarelli@vetanco.com.br, antonise.jaguezeski@gmail.com, gtellez@uark.edu,
\end{abstract} aleksandro_ss@yahoo.com.br

\section{RESUMEN}

El propósito del presente estudio fue determinar la frecuencia de la coccidiosis en pollos de engorde, detectar las especies más comunes de Eimeria spp y cargas parasitarias en aves comerciales en Brasil de 2012 a 2019. Estudiamos 13,648 aves entre 9 y 49 días de edad en 82 empresas en 13 estados brasileños. Las aves se seleccionaron al azar ( 3 a 6 aves / aviario) y se sacrificaron por eutanasia. Se analizaron las lesiones macroscópicas y se raspó la mucosa intestinal para el conteo de ooquistes de E. maxima. La clasificación de especies se basó en el tamaño de los ooquistes. También cuantificamos la carga infecciosa mediante un sistema de puntuación. Se detectó coccidiosis subclínica ( $E$. maxima micro) en un promedio de $34.8 \%$. E. acervulina (16.1\%), E. maxima (7.9\%) y E. tenella (4.1\%) también se identificaron con fluctuaciones anuales. El conteo de ooquistes estuvo dentro de la puntuación 1 (1 a 10 ooquistes / ave) en el 47.5 al $84.4 \%$ de los casos; seguido de una puntuación 2 ( 11 a 20), 3 (21 a 40) y 4 (más de 41 ooquistes / ave). La coccidiosis subclínica es motivo de preocupación, ya que 3.5 de cada diez aves estaban infectadas. Este es un factor responsable del menor rendimiento productivo de los pollos de engorde.

Palabras clave: Coccidiosis, Eimeria acervulina, Eimeria maxima, Eimeria tenella, prevalencia.

\begin{abstract}
The purpose of the present study was to determine the occurrence of coccidiosis in broilers, detect the most commonly occurring species of Eimeria spp, and parasitic burdens in industrial poultry in Brazil from 2012 to 2019. We studied 13,648 birds between 9-49 days of age at 82 companies in 13 Brazilian states. Birds were randomly selected (3-6 birds/aviary) and euthanized. Macroscopic lesions were analyzed, and the intestinal mucosa was scraped to count E. maxima oocysts. The classification of species was based on the size of the oocysts. We also quantified the infectious burden using a scoring system. Subclinical coccidiosis (E. maxima micro) was detected in an average of $34.8 \%$, E. acervulina $(16.1 \%)$, E. maxima $(7.9 \%)$, and E. tenella $(4.1 \%)$ were also identified with annual fluctuations. The count of oocysts was within score 1 (1-10 oocysts/bird) in $47.5-84.4 \%$ of the cases; followed by score 2 (11-20), 3 (21-40) and 4 (over 41 oocysts/bird). Subclinical coccidiosis is a cause for concern, with 3.5 out of every ten birds being infected. This is a factor responsible for the lower productive performance of broilers.
\end{abstract}

Keywords: Coccidiosis, Eimeria acervulina, Eimeria maxima, Eimeria tenella, prevalence. 


\section{INTRODUCCIÓN}

En la cría de aves de corral comercial e intensiva, Eimeria spp., son un protozoo ubicuo, muy extendido en seis continentes (Chapman et al., 2016; Clark et al., 2016). Se han reconocido siete especies de Eimeria (E. acervulina, E. brunetti, E. maxima, E. mitis, E. necatrix, E. praecox y E. tenella). Se diferencian en patogenicidad (McDougald, 2008). Las aves infectadas por Eimeria pueden reducir el rendimiento del crecimiento a través de la función intestinal deteriorada (Kim et al., 2017; Lu et al., 2019). Más comúnmente, las aves permanecen asintomáticas hasta que hay una infección por un gran número de coccidios o alguna otra patología agravante (Williams, 2005; Chapman et al., 2016; Gazoni et al., 2017). A principios de la década de 2000 se estimó que la enfermedad tenía un impacto económico anual de aproximadamente US \$ 3 mil millones, con pérdidas para los productores y la industria avícola mundial (Dalloul y Lillehoj, 2006). En Rumanía, en 2016, los investigadores encontraron que las pérdidas económicas totales por cada 24 manadas de 18.000 pollitos fueron de aproximadamente $37.948,2 €$, con una media de $3.162,4 €$ por manada. Estas pérdidas fueron causadas por la mortalidad $(34,8 \%)$ y la mala conversión alimenticia (65,2\%) debido a la coccidiosis (Györke et al., 2016).

Tradicionalmente, el diagnóstico de las granjas se da a través de la detección y enumeración de ooquistes excretados en las heces, además de medir las dimensiones de las aves. Los investigadores post-mortem evalúan la porción intestinal afectada y las lesiones (Long y Joyner, 1984). El diagnóstico específico de infecciones por Eimeria en pollos de engorde es fundamental para una mejor comprensión de la epidemiología y dinámica de la enfermedad y es necesario para la prevención, vigilancia y control efectivo (Morris and Gasser, 2006; Gazoni et al., 2017).

La coccidiosis subclínica se observa comúnmente en granjas avícolas en Brasil, y un diagnóstico preciso es esencial para rastrear las intervenciones, principalmente debido a problemas con la resistencia a los agentes coccidiostáticos (Gazoni et al., 2020). Por lo tanto, el objetivo de este estudio fue determinar la ocurrencia anual de la infección por Eimeria en pollos de engorde industriales de 2012 a 2019 en Brazil.

\section{MATERIAL Y MÉTODOS}

\section{Animales y recopilación de datos}

El seguimiento de la coccidiosis se llevó a cabo en 82 empresas de los estados de Rio Grande do Sul, Santa Catarina, Paraná, Mato Grosso do Sul, São Paulo, Minas Gerais, Rio de Janeiro, Goiás, Distrito Federal, Alagoas, Para, Paraiba y Pernambuco de 2012 y 2019, utilizando 13,648 pollos de engorde. Brasil es un país continental con una variación climática muy particular para cada estado, por lo que no se planteó en consideración de información climática. Los datos fueron divulgados por el Programa de Salud Intestinal (PSI) de Vetanco do Brasil, con el fin de obtener el porcentaje de aves afectadas y su grado de clasificación por puntajes de lesión en colaboración con investigadores de la Universidad Estatal de Santa Catarina (UDESC). Las aves eran 
de las líneas Cobb 500 y Aviagen y tenían entre 9 y 49 días de edad. Se les suministró piensos preparados por sus respectivas empresas, sin ninguna interferencia del evaluador en términos de formulación o uso de potenciadores del rendimiento y agentes anticoccidiales. El programa anticoccidial más utilizado es el sistema dual, en el que se utiliza un ingrediente activo en la primera fase (del 1ํal $21^{\circ}$ día de edad) y otro en la segunda fase (del $22^{\circ}$ día a 3 días antes del sacrificio), los pollos de engorde No recibir programas de vacunas contra la coccidiosis, con el fin de reducir la posibilidad de aparición de resistencias antimicrobianas.

\section{Colección de muestra}

Monitoreamos la salud intestinal de los pollos de engorde usando de 3 a 6 aves por lote (aviario). Las aves se seleccionaron al azar de tres puntos diferentes en el aviario (entrada, medio y fondo). Estas aves fueron sacrificadas por dislocación cervical, seguida de disección para recolección de muestras y evaluación visual.

\section{Evaluación macroscópica de tejidos}

En el tracto gastrointestinal se observó la presencia de lesiones causadas por $E$. acervulina, E. maxima y E. tenella, por ejemplo, figura 1. Cuando estaban presentes, las lesiones se clasificaron según su grado de intensidad, según la metodología de Johnson y Reid_(1970), donde la puntuación 0 indica la ausencia de lesión y la puntuación 4 indica daño severo.

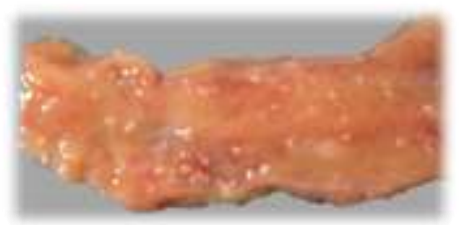

Lesiones E. acervulina

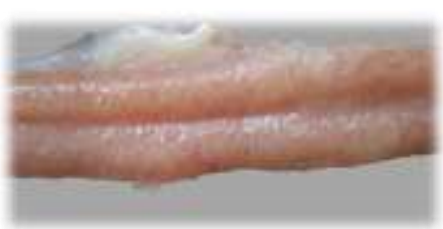

Lesiones E. maxima

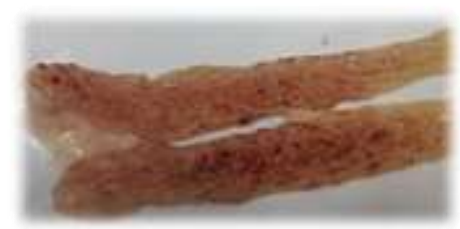

Lesiones E. tenella

Figura 1. Presencia de las lesiones causadas por E. acervulina, E. maxima, y E. tenella.

\section{Técnica de raspado de mucosas y recuento de ooquistes}

Para evaluar E. maxima micro, se utilizó la técnica de raspado de la mucosa intestinal para el recuento de ooquistes, realizada en la porción intestinal que rodea el divertículo de Meckel. El contenido fecal se depositó en un portaobjetos, se cubrió con un cubreobjetos y se visualizó en cinco campos (extremidades y centro) para contar ooquistes bajo microscopía óptica (100x). Se utilizaron cuatro puntajes para la clasificación, según Vetanco do Brasil (2011), donde el puntaje 0 representó ausencia de ooquistes; la puntuación 1 representó de 1 a 10 ooquistes; la puntuación 2 representó 11-20 ooquistes; la puntuación 3 representó 21-40 ooquistes, y la puntuación 4 significó más de 41 ooquistes, por ejemplo la figura 2. 
Puntuación 1 representado $1-10$ ooquistes

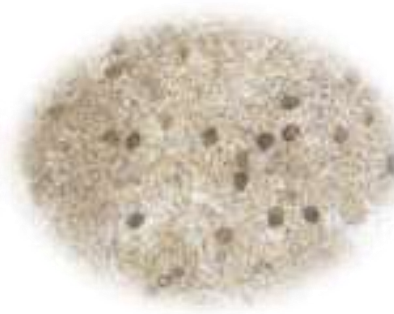

\section{Puntuación 2} representado $11-20$ ooquistes

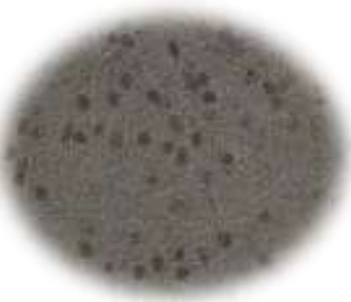

Puntuación 3 representado $21-40$ ooquistes

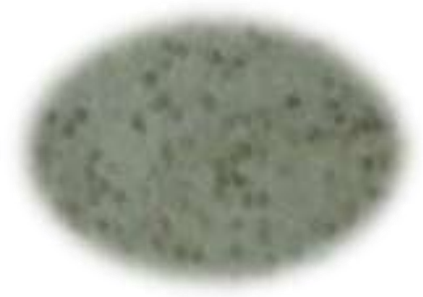

Puntuación 4 significa más de 41 ooquistes

Figura 2. Se utilizaron cuatro puntajes para la clasificación de E. maxima micro

\section{RESULTADOS}

La ocurrencia anual mostró diferencias significativas entre especies; mientras que uno era alto, los otros eran más pequeños en el año en cuestión. La coccidiosis subclínica tuvo la mayor incidencia (promedio de 34,8\%) durante el período evaluado (20122019), revelando una mayor frecuencia y diseminación en la industria avícola brasileña. Para las lesiones macroscópicas atribuidas a E. acervulina, E. maxima y $E$. tenella, la ocurrencia promedio fue de 16,1\%,7,9\% y 4,1\%, respectivamente. También observamos que ninguna de las especies de Eimeria mostró comportamiento lineal; en cambio, demostraron fluctuaciones a lo largo de los años: a veces, hubo un aumento. A veces, hubo una disminución en la ocurrencia (Tabla 1).

Considerando cada especie a través de períodos, observamos que, en 2012, las ocurrencias de E. maxima (22.2\%) y E. tenella (10.0\%) fueron mayores y menores en los otros años. E. acervulina tuvo un aumento significativo en 2016, alcanzando el $30,5 \%$ de ocurrencia, con una disminución gradual a partir de esa fecha $(9,7 \%$ en 2019). E. maxima micro mostró un crecimiento casi incremental hasta 2017 (del 28,8 al $45,5 \%)$, manteniéndose con un alto porcentaje anual en los años posteriores $(38,0-$ 42,6\%). Estos resultados se muestran en la Tabla 1.

Cuadro 1. Especies de Eimeria y porcentaje de eimeriosis en pollos de engorde entre 2012 a 2019

\begin{tabular}{llcccccccc}
\hline \multirow{2}{*}{ Especies } & \multicolumn{8}{c}{ Ocurrencia anual (\%) } \\
\cline { 2 - 10 } & 2012 & 2013 & 2014 & 2015 & 2016 & 2017 & 2018 & 2019 & Average \\
\hline E. acervulina & 9.3 & 18.7 & 13.6 & 13.5 & 30.5 & 16.6 & 17.1 & 9.7 & 16.1 \\
E. maxima & 22.2 & 7.8 & 4.8 & 5.9 & 8.1 & 4.4 & 4.9 & 5.4 & 7.9 \\
E. tenella & 10.0 & 5.0 & 4.1 & 2.0 & 4.5 & 2.3 & 1.7 & 3.1 & 4.1 \\
E. maxima, micro & 28.8 & 29.1 & 25.8 & 33.5 & 34.7 & 45.5 & 38.0 & 42.6 & 34.8 \\
\hline
\end{tabular}

En cuanto a las puntuaciones de las lesiones, en todos los años prácticamente y para todas las especies, el recuento de ooquistes estuvo dentro de la puntuación 1, seguido de las puntuaciones 2, 3 y 4 (rara vez fue superior a 3, y cuando ocurrió, se observó para $E$.máxima micro). La puntuación 4 fue poco frecuente, lo que a menudo resulta en un valor nulo. El porcentaje entre especies y entre años no siguió un patrón. En cambio, varió para ambas especies y año (Tabla 2). 
Cuadro 2. Porcentaje de puntajes de coccidiosis (Eimeria) obtenidos entre 2012 y 2019 en pollos de engorde de 9 a 49 días en la agroindustria brasileña

\begin{tabular}{|c|c|c|c|c|c|}
\hline \multirow{2}{*}{ Año } & \multirow{2}{*}{ Especies } & \multicolumn{4}{|c|}{ Puntuación (\%) } \\
\hline & & 1 & 2 & 3 & 4 \\
\hline \multirow{4}{*}{2012} & E. acervulina & 48.7 & 39.5 & 11.8 & 0.0 \\
\hline & E. maxima & 56.1 & 35.6 & 7.2 & 1.1 \\
\hline & E. tenella & 79.1 & 17.2 & 1.8 & 1.8 \\
\hline & E. maxima, micro & 73.0 & 9.8 & 9.4 & 7.9 \\
\hline \multirow{4}{*}{2013} & E. acervulina & 57.0 & 32.9 & 9.0 & 1.1 \\
\hline & E. maxima & 69.1 & 24.1 & 5.8 & 1.0 \\
\hline & E. tenella & 84.4 & 12.3 & 3.3 & 0.0 \\
\hline & E. maxima, micro & 58.8 & 14.4 & 7.8 & 19.0 \\
\hline \multirow{4}{*}{2014} & E. acervulina & 65.9 & 26.5 & 7.6 & 0.0 \\
\hline & E. maxima & 64.7 & 25.2 & 5.9 & 4.2 \\
\hline & E. tenella & 76.5 & 19.6 & 3.9 & 0.0 \\
\hline & E. maxima, micro & 65.3 & 14.6 & 4.2 & 15.9 \\
\hline \multirow{4}{*}{2015} & E. acervulina & 62.2 & 27.4 & 8.9 & 1.5 \\
\hline & E. maxima & 65.8 & 30.7 & 3.5 & 0.0 \\
\hline & E. tenella & 69.2 & 17.9 & 12.8 & 0.0 \\
\hline & E. maxima, micro & 76.2 & 12.8 & 2.5 & 8.6 \\
\hline \multirow{4}{*}{2016} & E. acervulina & 51.0 & 28.4 & 18.6 & 2.0 \\
\hline & E. maxima & 59.3 & 33.3 & 3.7 & 3.7 \\
\hline & E. tenella & 66.7 & 13.3 & 13.3 & 6.7 \\
\hline & E. maxima, micro & 70.7 & 14.7 & 7.8 & 6.9 \\
\hline \multirow{4}{*}{2017} & E. acervulina & 81.1 & 13.8 & 5.1 & 0.0 \\
\hline & E. maxima & 79.5 & 15.1 & 5.5 & 0.0 \\
\hline & E. tenella & 57.9 & 31.6 & 10.5 & 0.0 \\
\hline & E. maxima, micro & 71.5 & 10.3 & 1.9 & 16.3 \\
\hline \multirow{4}{*}{2018} & E. acervulina & 53.6 & 41.2 & 5.2 & 0.0 \\
\hline & E. maxima & 66.7 & 28.3 & 5.0 & 0.0 \\
\hline & E. tenella & 76.2 & 23.8 & 0.0 & 0.0 \\
\hline & E. maxima, micro & 84.2 & 6.4 & 2.3 & 7.0 \\
\hline \multirow{4}{*}{2019} & E. acervulina & 75.8 & 18.7 & 5.5 & 0.0 \\
\hline & E. maxima & 66.7 & 29.4 & 3.9 & 0.0 \\
\hline & E. tenella & 65.5 & 34.5 & 0.0 & 0.0 \\
\hline & E. maxima, micro & 47.5 & 22.5 & 2.5 & 27.5 \\
\hline
\end{tabular}

\section{DISCUSIÓN}

Reportamos la ocurrencia de coccidiosis en la industria avícola brasileña por más de siete años. Los estudios que informan las características epidemiológicas de la coccidiosis en Brasil son raros. Es importante prestar atención a las especies que se encuentran en el territorio; nuestros hallazgos concuerdan con los de otros autores que reportaron frecuentemente E. acervulina, E. maxima y E. tenella (Moraes et al., 2015; Chapman et al., 2016; Kim et al., 2017); estas especies, además de ser 
reportadas con mayor frecuencia, son las que comúnmente desarrollan resistencia a las drogas sintéticas (Shivaramaiah et al., 2014).

Todas las especies de Eimeria están muy extendidas en los seis continentes. Sin embargo, puede haber una división regional en la diversidad genética y la estructura poblacional de las especies (Prakashbabu et al., 2017); o incluso, como describen Clark et al. (2016), puede haber variantes genéticas entre los hemisferios sur y norte, lo que representa un riesgo para la seguridad alimentaria y el bienestar animal si se propaga a áreas previamente ausentes. Se cree que esta variación es atribuible al uso de medicamentos y vacunas anticoccidiales (Prakashbabu et al., 2017).

Un estudio sobre la prevalencia de coccidiosis en Santa Catarina (BR) mediante PCR mostró que el $96 \%$ de las fincas fueron positivas para Eimeria, con siete especies identificadas: E. maxima (63,7\%) y E. acervulina (63,3\%), E. tenella (54,6\%), E. mitis $(38,6 \%)$, E. praecox $(25,1 \%)$, E. necatrix $(24,3 \%)$ y E. brunetti $(13,1 \%)$, con un promedio de 2,96 especies por explotación (Moraes et al., 2015). Otro estudio en una pequeña región del estado de Tocantins reportó la ocurrencia de coccidiosis en todas las granjas, con la presencia de E. maxima, E. acervulina, E. mitis y E. tenella (Toledo et al., 2011). La alta preponderancia de una especie de Eimeria puede indicar su resistencia a los fármacos habitualmente expuestos, como es el caso de E. tenella estudiado en Nigeria (Ojimelukwe et al., 2018) E. maxima micro y E. acervulina en el presente estudio y el otros informaron en Brasil. El diagnóstico es importante y necesario para delinear estrategias, según Teeter et al. (2008), se observó que por cada punto de aumento en la evaluación microscópica de las lesiones de coccidiosis, el aumento de peso diario disminuyó un 1,5\% del peso corporal (g) durante el período de desafío de seis días. Por tanto, es fundamental conocer el grado de daño intestinal para cuantificar el rendimiento de las aves.

El ciclo de replicación intestinal rápido (4 a 6 días) y la vía oral/fecal hacen de la coccidiosis un problema severo de reproducción intensiva, causado por infecciones recurrentes y resistencia a agentes coccidiostáticos (Shivaramaiah et al., 2014). Sin un control efectivo, el número de parásitos puede aumentar hasta el punto de producir coccidiosis clínica. Para evitar resistencias, se llevan a cabo programas de rotación de fármacos y vacunación (Lan et al., 2017). La rotación de agentes coccidiostáticos puede ayudar a esclarecer las oscilaciones significativas de ocurrencia entre especies de Eimeria, como se verifica en este estudio.

La vacunación es la medida profiláctica más interesante; sin embargo, solo se utiliza en reproductoras de pollos de engorde (Abdul Rasheed y Matsler, 2020) y gallinas ponedoras (Chapman et al., 2014). La vacunación comercial in ovo contra la coccidiosis en pollos de engorde se ha vuelto ampliamente aceptada en la industria avícola de EE. UU sin embargo, sus efectos sobre el rendimiento aún no se han estudiado (Sokale et al., 2020). Es fundamental enfatizar la importancia de los estudios de prevalencia de especies para el desarrollo de vacunas regionales, como en este estudio. Se ha analizado el uso de antimicrobianos con miras a reemplazarlos para evitar el problema de resistencias y se han propuesto varias alternativas (Kim et 
al., 2017; Bortoluzzi et al., 2019; Lu et al., 2019; Park et al., 2020). La baja cantidad de ooquistes presentes en las muestras de este estudio (puntuación 1) y las pocas observaciones de la puntuación cero (sin ooquistes/muestra) pueden indicar la eficacia del uso estratégico de anticoccidiales químicos a través de piensos.

Además de la pérdida de rendimiento, la coccidiosis es un factor predisponente para una patología aún más dañina en las aves, como la enteritis necrótica (Williams, 2005; Adhikari et al., 2020). La presencia de E. maxima fue considerada por Paiva y McElroy (2014) como un factor de riesgo esencial para la promoción de Clostridium perfringens, y esto debe ser considerado en la búsqueda de métodos de control efectivos para las granjas en Brasil.

Para el monitoreo del rebaño, la técnica de visualización de mucosas y recuento de ooquistes y clasificación sigue siendo la más viable para la agroindustria brasileña, en detrimento de la prueba qPCR, también disponible para diagnóstico y cuantificación (Velkers et al., 2010). Esto aumentaría considerablemente el seguimiento y requeriría un cuidado diferencial de las muestras.

\section{CONCLUSIONES}

En conclusión, la coccidiosis subclínica tiene una prevalencia preocupante, ya que 3,5 de cada diez aves tienen coccidiosis. Este es uno de los factores que probablemente causa reducciones en el desempeño productivo de los lotes de pollos de engorde, además de ser un factor predisponente para la clostridiosis. Debido a que los intentos de erradicar el parásito mediante cuarentena, desinfección y saneamiento no han tenido éxito (McDougald, 2008), es de fundamental importancia que los profesionales del sector avícola realicen evaluaciones subclínicas de coccidiosis de manera rutinaria, pudiendo así intervenir si es necesario, para mantener el desempeño productivo de los pollos de engorde.

\section{LITERATURA CITADA}

ABDUL RASHEED MS, Matsler PL. 2020. Assessment of protection against Eimeria tenella in broiler breeders conferred by a live anticoccidial vaccine and effects of vaccination on early pullet growth. Journal Applied of Poultry Research. 29(2):447-454. ISSN: 1056-6171. https://doi.org/10.1016/j.japr.2020.02.002

ADHIKARI P, Kiess A, Adhikari R, Jha R. 2020. An approach to alternative strategies to control avian coccidiosis and necrotic enteritis. Journal Applied of Poultry Research. 29(2):515-534. ISSN: 1056-6171. https://doi.org/10.1016/j.japr.2019.11.005

BORTOLUZZI C, Fernandes JIM, Doranalli K, Applegate TJ. 2019. Effects of dietary amino acids in ameliorating intestinal function during enteric challenges in broiler chickens. Animal Feed Science and Technology. 262:114383. ISSN: 0377-8401. https://doi.org/10.1016/j.anifeedsci.2019.114383 
CHAPMAN HD, Jeffers TK. 2014. Vaccination of chickens against coccidiosis ameliorates drug resistance in commercial poultry production. International Journal for Parasitology: Drugs and Drug Resistance. 4(3):214-217. ISSN: 0020-7519. https://doi.org/10.1016/j.ijpddr.2014.10.002

CHAPMAN HD, Barta JR, Hafeez MA, Matsler P, Rathinam T, Raccoursier M. 2016. The epizootiology of Eimeria infections in commercial broiler chickens where anticoccidial drug programs were employed in six successive flocks to control coccidiosis. Poultry Science. 95(8):1774-1778. ISSN: 0032-5791. https://doi.org/10.3382/ps/pew091

CLARK EL, Macdonald SE, Thenmozhi V, Kundu K, Garg G, Kumar S, Nolan MJ, Sudhakar NR, Adebambo AO, Lawal IA, Álvarez Zapata R, Awuni JA, Chapman HD, Karimuribo E, Mugasa CM, Namangala B, Rushton J, Sao X, Kumarasamy Thangaraj, Srinivasa Rao AS, Tewari AK, Banerjee PS, Raj GD, Raman M, Tomley FM, Blake DP. 2016. Cryptic Eimeria genotypes are common across the southern but not northern hemisphere. International Journal for Parasitology. 46(9):537-544. ISSN: 0020-7519. https://doi.org/10.1016/j.ijpara.2016.05.006

DALLOUL RA, Lillehoj HS. 2006. Poultry coccidiosis: recent advancements in control measures and vaccine development. Expert Review of Vaccines. 2006(5):143-163. ISSN: 1476-0584. https://doi.org/10.1586/14760584.5.1.143

GAZONI FL, Adorno FC, Matte F, Malta T, Felin MR, Urbano T, Zampar A, HernandezVelasco X, Tellez G. 2017. Study of the correlation between intestinal health and prevalence of coccidiosis in broiler chickens of brazilian agribusinesses between the years 2015 and 2016. International Journal of Poultry Science. 16(10):381-386. ISSN: 1682-8356. https://doi.org/10.3923/ijps.2017.381.386

GAZONI FL, Adorno FC, Matte F, Alves AJ, Campagnoni IDP, Urbano T, da Silva AS. 2020. Correlation between intestinal health and coccidiosis prevalence in broilers in Brazilian agroindustries. Parasitology International. 76:102027. ISSN: 1383-5769. https://doi.org/10.1016/j.parint.2019.102027

GYÖRKE A, Kalmár Z, Pop, LM, Şuteu OL. 2016. The economic impact of infection with Eimeria spp. in broiler farms from Romania. Revista Brasileira de Zootecnia. 45(5):273-280. ISSN: 1806-9290. https://doi.org/10.1590/S1806-92902016000500010

JOHNSON J, Reid WM. 1970. Anticoccidial Drugs: lesion scoring techniques in battery and floor-pen experiments with chickens. Experimental Parasitology. 28:30-36. ISSN: 0014-4894. https://doi.org/10.1016/0014-4894(70)90063-9

KIM E, Leung H, Akhtar N, Li J, Barta JR, Wang Y, Kiarie E. 2017. Growth performance and gastrointestinal responses of broiler chickens fed corn-soybean meal diet without 
or with exogenous epidermal growth factor upon challenge with Eimeria. Poultry Science. 96(10):3676-3686. ISSN: 0032-5791. https://doi.org/10.3382/ps/pex192

LAN LH, Sun B.B., Zuo BXZ, Chen XQ, Du AF. 2017. Prevalence and drug resistance of avian Eimeria species in broiler chicken farms of Zhejiang province, China. Poultry Science. 96(7):2104-2109. ISSN: 0032-5791. https://doi.org/10.3382/ps/pew499

LONG PL, Joyner LP. 1984. Problems in the identification of species of Eimeria. The Journal of Protozoology. 31(4):535-541. ISSN:1550-7408. https://doi.org/10.1111/j.1550-7408.1984.tb05498.x

LU Z, Thanabalan A, Leung H, Akbari Moghaddam Kakhki R, Patterson R, Kiarie EG. 2019. The effects of feeding yeast bioactives to broiler breeders and/or their offspring on growth performance, gut development, and immune function in broiler chickens challenged with Eimeria. Poultry Science. 98(12):6411-6421. ISSN: 0032-5791. https://doi.org/10.3382/ps/pez479

MCDOUGALD LR. 2008. "Protozoal infections". In: Saif YM, Fadly AM, Glisson JR, McDougald LR, Nolan LK, Swayne DE. Editors. Diseases of Poultry. 12th ed. Ames, lowa, Blackwell Publishing. Pp. 1067. ISBN-13:978-0-8138-0718-8

MORAES JC, França M, Sartor AA, Bellato V, de Moura AB, Magalhães MDLB, Miletti LC. 2015. Prevalence of Eimeria spp. in broilers by multiplex PCR in the southern region of Brazil on two hundred and fifty farms. Avian Diseases. 59(2):277-281. ISSN: 0005-2086. https://doi.org/10.1637/10989-112014-Reg

MORRIS GM, Gasser RB. 2006. Biotechnological advances in the diagnosis of avian coccidiosis and the analysis of genetic variation in Eimeria. Biotechnology Advances. 24(6):590-603. ISSN: 0734-9750. https://doi.org/10.1016/j.biotechadv.2006.06.001

OJIMELUKWE AE, Emedhem DE, Agu GO, Nduka FO, Abah AE. 2018. Populations of Eimeria tenella express resistance to commonly used anticoccidial drugs in southern Nigeria. International Journal of Veterinary Science and Medicine. 6(2):192-200. ISSN: 2314-4599. https://doi.org/10.1016/j.jjvsm.2018.06.003

PAIVA D, McElroy A. 2014. Necrotic enteritis: applications for the poultry industry. Journal Applied of Poultry Research. 23:557-566. ISSN: 1056-6171. https://doi.org/10.3382/japr.2013-00925

PARK I, Lee Y, Goo D, Zimmerman NP, Smith AH, Rehberger T, Lillehoj HS. 2020. The effects of dietary Bacillus subtilis supplementation, as an alternative to antibiotics, on growth performance, intestinal immunity, and epithelial barrier integrity in broiler chickens infected with Eimeria maxima. Poultry Science. 99(2):725-733. ISSN: 00325791. https://doi.org/10.1016/j.psj.2019.12.002 
PRAKASHBABU BC, Thenmozhi V, Limon G, Kundu K, Kumar S, Garg R, Banerjee PS, Clark EL, Srinivasa Rao ASR, Raj DG, Raman M, Banerjee PS, Tomley FM, Guitian J, Blake DP. 2017. Eimeria species occurrence varies between geographic regions and poultry production systems and may influence parasite genetic diversity. Veterinary Parasitology. 233:62-72. ISSN: 1873-2550. https://doi.org/10.1016/j.vetpar.2016.12.003

SHIVARAMAIAH C, Barta RJ, Hernandez-Velasco X, Tellez G, Hargis BM. 2014. Coccidiosis: recent advancements in the immunobiology of Eimeria species, preventive measures, and the importance of vaccination as a control tool against these Apicomplexan parasites. Veterinary Medicine. 5:23-34. ISSN: 2230-2034. https://doi.org/10.2147/VMRR.S57839

SOKALE AO, Williams CJ, Triplett MD, Hoerr FJ, Peebles ED. 2020. Effects of stage of broiler embryo development on coccidiosis vaccine injection accuracy, and subsequent oocyst localization and hatchling quality. Poultry Science. 99(1):189-195. ISSN: 0032-5791. https://doi.org/10.3382/ps/pez592

TEETER RG, Beker A, Brown C, Broussard C, Fitz-Coy S, Radu J, Newman L. (2008). Transforming coccidiosis-mediated lesion scores into production and calorific cost. Proc. 23rd World Poult. Congr., Brisbane, Australia. 29.06.2008-04.07.2008. Pp.1821. https://wenku.baidu.com/view/9df3d94fe45c3b3567ec8ba4.html

TOLEDO GA, Almeida JDDM, Almeida KDS, Freitas FLDC. 2011. Coccidiosis in broiler chickens raised in the Araguaína region, State of Tocantins, Brazil. Revista Brasileira de Parasitologia Veterinaria. 20(3):249-252. ISSN 0103-846X. http://dx.doi.org/10.1590/S1984-29612011000300014

VELKERS FC, Blake DP, Graat EAM, Vernooij JCM, Bouma A, De Jong MCM Stegeman JA. 2010. Quantification of Eimeria acervulina in faeces of broilers: Comparison of McMaster oocyst counts from $24 \mathrm{~h}$ faecal collections and single droppings to real-time PCR from cloacal swabs. Veterinary Parasitology. 169(1-2):17. ISSN: 0304-4017. https://doi.org/10.1016/j.vetpar.2010.01.001

VETANCO do Brasil. 2011. Programa de Saúde Intestinal. Padrão microscópio de escore para E. maxima. Pp. 20 -21. https://www.vetanco.com/br/publicacoes-tecnicas/

WILLIAMS RB. 2005. Intercurrent coccidiosis and necrotic enteritis of chickens: rational, integrated disease management by maintenance of gut integrity. Avian Pathology. 34(3):159-180. ISSN: 0307-9457.

https://doi.org/10.1080/03079450500112195 